\title{
Kinetics of Conformational Sampling in Ubiquitin**
}

\author{
David Ban, Michael Funk, Rudolf Gulich, Dalia Egger, T. Michael Sabo, Korvin F. A. Walter, \\ R. Bryn Fenwick, Karin Giller, Fabio Pichierri, Bert L. de Groot, Oliver F. Lange, \\ Helmut Grubmüller, Xavier Salvatella, Martin Wolf, Alois Loidl, Reiner Kree,* Stefan Becker, \\ Nils-Alexander Lakomek, * Donghan Lee,* Peter Lunkenheimer,* and Christian Griesinger*
}

Molecular recognition plays a central role in many biological processes. For enzymatic reactions and slow protein-protein recognition events, turn-over rates and on-rates in the millisecond-to-second time scale have been connected to internal protein dynamics detected with atomic resolution by NMR spectroscopy, and in particular conformational sampling could be established as a mechanism for enzyme-substrate and protein-protein recognition. ${ }^{[1-5]}$ Recent theoretical studies indicate that faster rates of conformational interconversion in the microsecond time scale might limit on-rates for protein-protein recognition. ${ }^{[6,7]}$ However experimental proofs were lacking so far, mainly because such rates could not be determined accurately enough and kinetic experiments in the microsecond time range are difficult to perform.

Nevertheless, for proteins and TAR-RNA, ${ }^{[8-10]}$ recent studies based on residual dipolar couplings (RDCs) and other NMR spectroscopy techniques ${ }^{[11,12]}$ have detected substantial internal dynamics in a time window from the rotational correlation time $\tau_{\mathrm{c}}$ (one-digit nanoseconds) to approximately $50 \mu \mathrm{s},{ }^{[8,13-15]}$ called the supra- $\tau_{\mathrm{c}}$ window in the following. However, the exact rates of internal dynamics within this four orders of magnitude wide time window could not be determined.

Supra- $\tau_{\mathrm{c}}$ dynamics in ubiquitin ${ }^{[9]}$ and TAR-RNA ${ }^{[16]}$ could be connected to the conformational sampling required for

$\left[{ }^{*}\right]$ D. Ban, ${ }^{[+]}$M. Funk, ${ }^{[+]}$Dr. T. M. Sabo, K. F. A. Walter, K. Giller, Dr. N.-A. Lakomek, Dr. D. Lee, Prof. Dr. C. Griesinger Department of NMR-based Structural Biology, Max Planck Institute for Biophysical Chemistry

Am Fassberg 11, 37077 Göttingen (Germany)

E-mail: lakomekn@niddk.nih.gov dole@nmr.mpibpc.mpg.de cigr@nmr.mpibpc.mpg.de

R. Gulich, D. Egger, M. Wolf, Prof. Dr. A. Loidl, Dr. P. Lunkenheimer Experimental Physics V, University of Augsburg

Universitätsstraße 2, 86135 Augsburg (Germany)

E-mail: peter.lunkenheimer@physik.uni-augsburg.de

Prof. Dr. R. Kree

Department of Theoretical Physics, University of Göttingen

Friedrich-Hund-Platz 1, 37077 Göttingen (Germany)

E-mail: kree@theorie.physik.uni-goettingen.de

R. B. Fenwick, Dr. X. Salvatella

Laboratory of Molecular Biophysics

ICREA and Institute for Research in Biomedicine Barcelona

Parc Cientific de Barcelona C/Baldiri Reixac 10

08028 Barcelona (Spain)

Dr. F. Pichierri

G-COE Laboratory, Department of Applied Chemistry

Tohoku University, 980-8579, Sendai (Japan) molecular recognition. While the amplitudes of motions have been indirectly detected by RDCs and characterized in great detail, it has so far been impossible to directly observe these motions and to determine the exact rate of these supra- $\tau_{\mathrm{c}}$ motions. In contrast, conformational sampling in enzymes occurs on a time scale that is 100 to 1000 times slower than supra- $\tau_{\mathrm{c}}$ dynamics and therefore NMR relaxation dispersion (RD) techniques have been able to establish the functional link to enzyme kinetics with atomic resolution at physiological conditions. ${ }^{[1,2,5]}$ However, for technical reasons, RD is not sensitive to motion faster than approximately $50 \mu \mathrm{s}$ (RD window) and therefore does not access motion in the supra- $\tau_{\mathrm{c}}$ window at room temperature.

Here we determine the rate of interconversion between conformers of free ubiquitin by a combination of NMR RD experiments in super-cooled solution and dielectric relaxation spectroscopy (DR). Furthermore, we corroborate the motional amplitudes in the RDC-derived ensembles quantitatively with the observed amplitudes of RD and DR. The methods utilized herein can be used to directly study protein dynamics in a time range that was previously inaccessible.

Significant motional amplitude in the supra- $\tau_{\mathrm{c}}$ window has been observed using RDC measurements, and was connected to the conformational sampling for a protein in the ground

Dr. B. L. de Groot, O. F. Lange, Prof. Dr. H. Grubmüller Department of Theoretical and Computational Biophysics Max Planck Institute for Biophysical Chemistry Am Fassberg 11, 37077 Göttingen (Germany)

$\left[{ }^{+}\right]$These authors contributed equally.

[***] This work was supported by the Max Planck Society (grants to C.G., B.L.d.G., and H.G.), the Fonds der Chemischen Industrie, the German Israel Funds, the DFG, the ERC (ERC grant agreement number 233227 to C.G.), the Ministerio de Ciencia e Innovación (MICINN) and the Institute for Research in Biomedicine Barcelona (IRB) (grant to X.S.), the Global COE Program of Tohoku University and the Japan Society for the Promotion of Science (JSPS; grant to F.P.). T.M.S. thanks for a Alexander-von-Humboldt Fellowship. We are grateful to Konrad Samwer, Physics Department, University of Göttingen, for pointing out the power of dielectric relaxation spectroscopy, to Dietmar Pörschke, Max Planck Institute for Biophysical Chemistry, Göttingen, for discussions about dielectric spectroscopy and sample preparation, to Edward d'Auvergne for discussions about NMR relaxation dispersion, to Thomas Szyperski (University at Buffalo, NY) and Hai-Young Kim for protocols and help, respectively, for the preparation of NMR samples in supercooled solutions, and to Markus Zweckstetter for general discussions.

Dupporting information for this article is available on the WWW under http://dx.doi.org/10.1002/anie.201105086. 
state.${ }^{[9]}$ It was also shown that this conformational sampling is required for molecular recognition. ${ }^{[9]}$ In principle, the motional amplitude in the supra- $\tau_{\mathrm{c}}$ window should be detectable by RD measurements to give kinetic information. However, until now at room temperature, RD for ubiquitin was not observed except for amide nitrogens close to Glu24 that experience hydrogen bond dynamics on a millisecond time scale (see the Supporting Information). ${ }^{[17]}$ Motional processes in the supra- $\tau_{\mathrm{c}}$ window faster than $50 \mu$ s would therefore not be detected at room temperature because of limitations in the maximal radio frequency field that can be applied in the RD experiment. ${ }^{[18]}$ To overcome this limitation, we hypothesized that supra- $\tau_{\mathrm{c}}$ motion may shift into the RD sensitive time window by lowering the temperature because motions slow down at lower temperature. ${ }^{[17,19,20]}$ If we can detect the motions at several low temperatures, the extrapolation of conformational exchange time constants to room temperature should be possible by temperature-dependent measurements of the RD. Therefore, we measured RD data of ubiquitin in super-cooled water ${ }^{[20,21]}$ at temperatures between 265 and $277 \mathrm{~K}$ using an off-resonance $R_{1 \rho}$ sequence with TROSY read-out. ${ }^{[22]}$ We observed considerable RD for Ile13 and Val70 (Figure 1A,D), which play an important role in the molecular recognition of ubiquitin. For Ile13 and Val70
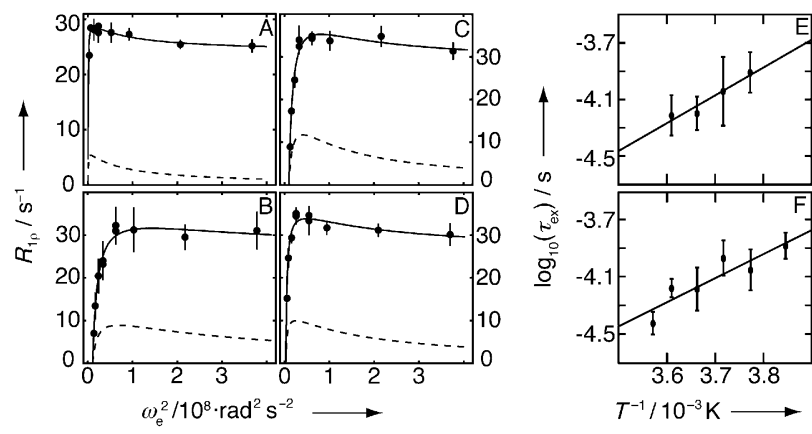

Figure 1. Temperature-dependent relaxation dispersion. $R_{1 \rho}$ dispersion curves measured at $-8^{\circ} \mathrm{C}$ for residues Ile13 (A), Ile23 (B), Asn25 (C), and $\mathrm{Val70}(\mathrm{D})$ plotted as a function of the rotating frame effective field. Solid and dashed lines represent the nonlinear fit to the data and the exchange contribution extracted from the fit, respectively. Arrhenius-type fit to the temperature-dependent RD exchange rates (see Table S1 in the Supporting Information) for Ile13 (E) and Val70 (F). Activation energies of $(37 \pm 7) \mathrm{kJ} \mathrm{mol}^{-1}$ and $(32 \pm 8) \mathrm{kJ} \mathrm{mol}^{-1}$ were extracted for $1 / \mathrm{e} 13$ and $\mathrm{Val} 70$, respectively.

at $265 \mathrm{~K}$, conformational exchange time constants of (122 \pm $40) \mu \mathrm{s}$ and $(90 \pm 30) \mu \mathrm{s}$, respectively, were measured (Table S1 in the Supporting Information). At $277 \mathrm{~K}$, life times shortened to $(61 \pm 20) \mu$ s and $(67 \pm 10) \mu$ s, respectively (Table S1 in the Supporting Information). Extrapolation of these life times to room temperature with the Arrhenius-type equation yields $(10 \pm 9) \mu$ s at $309 \mathrm{~K}$ (Figure $1 \mathrm{E}, \mathrm{F})$. The propagated error is of the same size as the average value because only a small temperature range shows RD. The determined rates agree well with the 3 to $30 \mu$ s backbone motion of the protein BPTI at room temperature in a recent extended molecular dynamics study. ${ }^{[23]}$
There are two further residues that show ${ }^{15} \mathrm{~N}$ relaxation dispersions, namely Ile23 and Asn25 (Figure 1B,C). Their relaxation dispersion was already observed at $280^{[17]}$ and $260 \mathrm{~K}^{[20]}$ and has been attributed to hydrogen-bond reordering, involving Thr55, with a population of an alternate conformation at approximately $2 \%$. This process is in the millisecond time window, ${ }^{[17]}$ which is several orders of magnitude slower than the microsecond motion described in this work. A more detailed discussion about this and other slow motion processes in ubiquitin is deferred to the Supporting Information. Other residues did not show relaxation dispersion within the current technical limit (see Figure S8 in the Supporting Information).

Relaxation dispersion experiments provide information about the rates but also the chemical shift variances induced by conformational sampling. Conversely, conformational ensembles can be used to determine the expected variance of the chemical shifts (see the Supporting Information) induced by conformational interconversions as long as all conformers within an ensemble can interconvert between each other. ${ }^{[24,25]}$ To investigate the supra- $\tau_{\mathrm{c}}$ time window $(<50 \mu \mathrm{s})$ three RDC-derived ensembles of ubiquitin in solution are available, namely the EROS, ${ }^{[9]}$ enhanced EROS, called henceforth EROSII, and the ERNST ensembles $^{[26]}$ (Figure 2A-I, see the Supporting Information for details on how the structural ensembles were determined). The two new ensembles agree equally well with the conformational selection scenario previously described for EROS. ${ }^{[9]}$ They are all ground-state ensembles of ubiquitin that do not contain excited states. So far relaxation dispersion has not been used to characterize the kinetics of interconversion for the ground state of a protein. The above-mentioned ensembles of ubiquitin are in the ground state and they do not contain information about the interconversion rates between the different equally populated members in the ensembles. Therefore, to estimate relaxation dispersion we simply

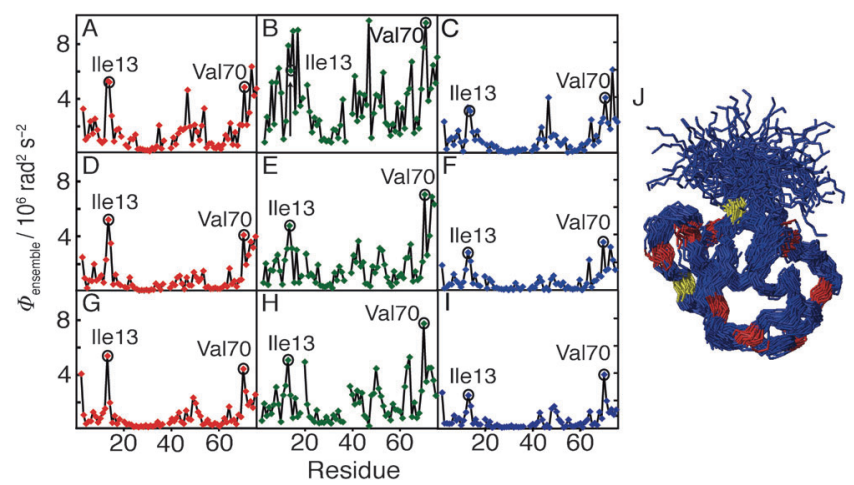

Figure 2. Predicted chemical shift variance $\left(\Phi_{\text {ensemble }}\right)$ of EROS (A, B, and $C)$, EROSII (D, E, and $F)$ and ERNST $(G, H$, and I) ensembles. Chemical shifts were extracted from the ensemble members $(N=116$ for EROS, $N=176$ for EROSII, $N=640$ for ERNST) with the programs SHIFTX (A, D, and G; red), SHIFTS (B, E, and H; green), and SPARTA (C, F, and I; blue). Residues Ile13 and Val70 showing consistently the largest $\Phi_{\text {ensemble }}$ through all ensembles and all programs are indicated. The EROS ensemble $(J)$ labelled with the RD detected residues, Ile13 and Val70 (yellow) and the eight residues with higher supra- $\tau_{\mathrm{c}}$ mobility (red). 
assume that all conformations in the ensemble interconvert with the same rate. This defines the maximum amount of relaxation dispersion that is compatible with the ensemble. If we assumed instead that certain conformations interconvert faster and others still slowly, this would not introduce additional residues expected to show experimental RD but rather reduce the number of residues predicted to show relaxation dispersion. All three RDC-based ensembles do not necessarily represent canonical ensembles, which however, is not required for the conclusions drawn in this work (see the Supporting Information).

Three programs were used to calculate chemical shifts from conformations: SHIFTS, SPARTA, and SHIFTX. ${ }^{[27-29]}$ Residues, for which the chemical shift variances $\left(\Phi_{\text {ensemble }}\right)$ were greater than the standard deviation from the average, were considered to be consistent. Only two residues, Ile13 and Val70, were above the cut-off in all independent calculations (Figure 2A-I). Even at two standard deviations from the average, only these two residues, Ile13 and Val70, fulfill this criterion, except only for one occasion for Ile13 when the EROS ensemble was calculated with the SHIFTS program (Figure 2B). Even there, Ile13 and Val70 are among the three residues with the largest chemical shift variation. The observation of the uniquely large chemical shift variation of Ile13 and Val70 did not change even if we randomly omitted half of the conformations in each of the ensembles. ${ }^{[17]}$

Despite the fact that Ile13 and Val70 show the largest chemical shift variance, there are eight backbone amides with higher mobility on the supra- $\tau_{\mathrm{c}}$ time scale (Figure $2 \mathrm{~J}$, lower ratio of the RDC-derived order parameter ${ }^{[30]}$ divided by the Lipari-Szabo order parameter: ${ }^{[31]} S_{\mathrm{RDC}}^{2} / S_{\mathrm{LS}}^{2}$ ), or faster dynamics than Val70, (see Figure S7 in the Supporting Information, for Ile13 there is no $S_{\mathrm{LS}}^{2}$ available) only Ile13 and Val70 show sufficient chemical shift variations such that RD is observed.

This highlights a connection between the microsecond interconversion time constant detected by RD and the impact of supra- $\tau_{\mathrm{c}}$ dynamics reflected through the structural variance between ubiquitin conformers in the ensembles. Thus, the RDC-derived ensembles that describe the amplitudes of conformational sampling correctly predict the NMR RD results in super-cooled solution at temperatures between 265 and $277 \mathrm{~K}$

To independently test the extrapolation of RD determined rates to room temperature and beyond we directly measured the rate of interconversion in the conformational ensemble at $309 \mathrm{~K}$ with dielectric relaxation (DR) spectroscopy in solution. This method can detect the frequencies of fluctuations of the bulk electric dipole moment of the solvent, solute (in our case ubiquitin), or of charge carriers, such as ions in a sample. As shown in Figure 3, for $309 \mathrm{~K}$, rotational diffusion of the static electrical dipole of ubiquitin gives rise to the well-known $\beta$ peak at $3 \tau_{c}^{\text {magnetic }}=\tau_{\beta}^{\text {electric }}=\left(2 \pi v_{\beta}^{\text {electric }}\right)^{-1}=11 \mathrm{~ns}$ which is in agreement with previous measurements ${ }^{[32]}$ (see the Supporting Information). In addition, a slower relaxation process within the supra- $\tau_{\mathrm{c}}$ window with a relaxation $\tau_{\text {sub- } \beta}$ centered around $1 \mu \mathrm{s}$ $\left(\log _{10} v_{\text {sub }-\beta} \approx 5\right.$ to 5.5$)$ was observed (Figure 3$)$, which we name the sub- $\beta$ peak. To our knowledge, sub- $\beta$ peaks in DR spectroscopy had previously not been observed in solution.

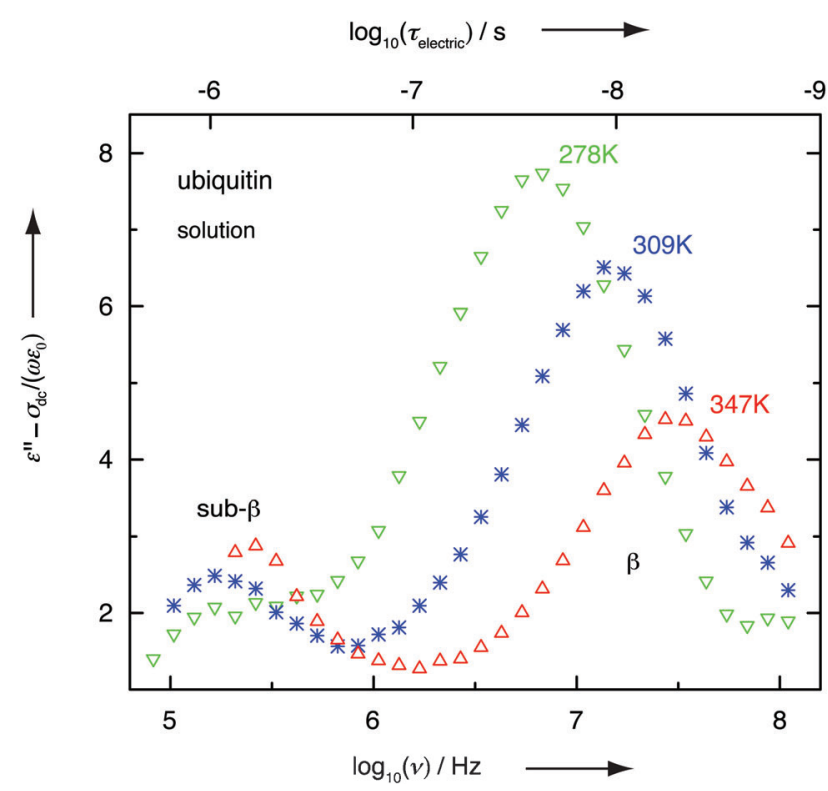

Figure 3. Dielectric relaxation measurements of ubiquitin in solution. Conductivity-corrected dielectric loss spectra of a $3 \mathrm{~mm}$ ubiquitin solution for three selected temperatures.

We explain the occurrence of this sub- $\beta$ peak with the fluctuation of the direct current (DC) induced by the interconversion between the different conformations of ubiquitin. According to this model, various conformations exhibit slightly different affinities to ions in aqueous solution, thus influencing the mobility of the ions that cause the DC in the DR spectrum (see the Supporting Information). In support of this model we reproduce the relative amplitude of the sub- $\beta$ peak (experimentally $1 \%$, theoretically for two extreme conformations $6 \%$ ) compared to the DC conductivity by computing with Poisson-Boltzmann continuum electrostatics calculations the variation of mobile ions around different members of the EROS ensemble ${ }^{[9]}$ (see the Supporting Information). In addition, the amplitude of this sub- $\beta$ peak was unchanged with the size of the electric field and it occurred independent of the concentration and the choice of the different ions (see the Supporting Information). At $309 \mathrm{~K}$ the motional process centered around $1 \mu$ s from DR agrees well with the RD extrapolated rates of 1 to $19 \mu$ s. From this evidence, we conclude that DR probes at room temperature the same motional process as RD at low temperature, namely the amplitude and kinetics of the conformational interconversion of ubiquitin.

Until now, relaxation dispersion has not been used to characterize the kinetics of interconversion for the ground state of a protein. However, through the use of super-cooled $\mathrm{RD}$, the conformational sampling rate for free ubiquitin has been directly measured at low temperature. Motional amplitudes from the RDC-derived ensembles, which embody supra- $\tau_{\mathrm{c}}$ dynamics, correctly predict the residues observed in the RD experiments (Ile13 and Val70) whereas classical excited states are not picked up by the RDC-derived ensembles because of the low population of the excited states (Ile23 and Asn25). In addition, DR independently identified the RD measured lifetime without extrapolation 
providing two independent techniques that observed the same supra- $\tau_{\mathrm{c}}$ motional process.

In summary, we have measured the rate of conformational interconversion for free ubiquitin with two methods. The results from both methods indicate that the major part of the supra- $\tau_{\mathrm{c}}$ motion in the ground state of ubiquitin occurs on a timescale of $(10 \pm 9) \mu \mathrm{s}$ at $309 \mathrm{~K}$, thus narrowing down the window from four orders of magnitude to one. In addition, we could predict chemical shift (relaxation dispersion) and ion flux (dielectric relaxation) variances from a ground-state ensemble which has not been done before. The combination of RDCs, NMR relaxation dispersion at low temperatures, and DR spectroscopy should be applicable for a wide range of systems and enable the functional investigation of protein dynamics in a previously hidden time window between nanoseconds and $50 \mu$ s.

Received: July 20, 2011

Published online: October 5, 2011

Keywords: dielectric spectroscopy $\cdot$ kinetics .

NMR spectroscopy $\cdot$ proteins $\cdot$ relaxation dispersion

[1] D. D. Boehr, D. McElheny, H. J. Dyson, P. E. Wright, Science 2006, 313, 1638 .

[2] E. Z. Eisenmesser, O. Millet, W. Labeikovsky, D. M. Korzhnev, M. Wolf-Watz, D. A. Bosco, J. J. Skalicky, L. E. Kay, D. Kern, Nature 2005, 438, 117.

[3] L. Leder, C. Berger, S. Bornhauser, H. Wendt, F. Ackermann, I. Jelesarov, H. R. Bosshard, Biochemistry 1995, 34, 16509.

[4] B. Ma, S. Kumar, C. J. Tsai, R. Nussinov, Protein Eng. 1999, 12, 713.

[5] L. Wang, Y. Pang, T. Holder, J. R. Brender, A. V. Kurochkin, E. R. Zuiderweg, Proc. Natl. Acad. Sci. USA 2001, 98, 7684.

[6] T. Wlodarski, B. Zagrovic, Proc. Natl. Acad. Sci. USA 2009, 106, 19346

[7] H. X. Zhou, Biophys. J. 2010, 98, L15.

[8] G. Bouvignies, P. Bernado, S. Meier, K. Cho, S. Grzesiek, R. Bruschweiler, M. Blackledge, Proc. Natl. Acad. Sci. USA 2005, $102,13885$.
[9] O. F. Lange, N. A. Lakomek, C. Fares, G. F. Schroder, K. F. A. Walter, S. Becker, J. Meiler, H. Grubmuller, C. Griesinger, B. L. de Groot, Science 2008, 320, 1471.

[10] Q. Zhang, X. Sun, E. D. Watt, H. M. Al-Hashimi, Science 2006, $311,653$.

[11] D. F. Hansen, H. Q. Feng, Z. Zhou, Y. W. Bai, L. E. Kay, J. Am. Chem. Soc. 2009, 131, 16257.

[12] B. Vögeli, T. F. Segawa, D. Leitz, A. Sobol, A. Choutko, D. Trzesniak, W. van Gunsteren, R. Riek, J. Am. Chem. Soc. 2009, 131,17215

[13] W. Peti, J. Meiler, R. Brueschweiler, C. Griesinger, J. Am. Chem. Soc. 2002, 124, 5822 .

[14] N. A. Lakomek, T. Carlomagno, S. Becker, J. Meiler, C. Griesinger, Angew. Chem. 2005, 117, 7954; Angew. Chem. Int. Ed. 2005, 44, 7776 .

[15] K. B. Briggman, J. R. Tolman, J. Am. Chem. Soc. 2003, 125, 10164.

[16] Q. Zhang, A. C. Stelzer, C. K. Fisher, H. M. Al-Hashimi, Nature 2007, 450, 1263.

[17] F. Massi, M. J. Grey, A. G. Palmer, Protein Sci. 2005, 14, 735.

[18] J. Cavanagh, J. F. Fairbrother, A. G. Palmer III, M. Rance, J. N. Skelton, Principles and Practice: Protein NMR Spectroscopy, 2nd ed., Elsevier Academic Press, 2007.

[19] J. Dittmer, G. Bodenhausen, J. Am. Chem. Soc. 2004, 126, 1314.

[20] J. L. Mills, T. Szyperski, J. Biomol. NMR 2002, 23, 63.

[21] J. J. Skalicky, D. K. Sukumaran, J. L. Mills, T. Szyperski, J. Am. Chem. Soc. 2000, 122, 3230.

[22] J. G. Kempf, J. Y. Jung, N. S. Sampson, J. P. Loria, J. Am. Chem. Soc. 2003, 125, 12064.

[23] D. E. Shaw, P. Maragakis, K. Lindorff-Larsen, S. Piana, R. O. Dror, M. P. Eastwood, J. A. Bank, J. M. Jumper, J. K. Salmon, Y. Shan, W. Wriggers, Science 2010, 330, 341.

[24] L. E. Kay, Nat. Struct. Biol. 1998, NMR supplement, 513.

[25] A. Mittermaier, L. E. Kay, Science 2006, 312, 224.

[26] R. B. Fenwick, S. Esteban-Martin, B. Richter, D. Lee, K. F. Walter, D. Milovanovic, S. Becker, N. Lakomek, C. Griesinger, X. Salvatella, J. Am. Chem. Soc. 2011, 133, 10336-10339.

[27] S. Neal, A. M. Nip, H. Zhang, D. S. Wishart, J. Biomol. NMR 2003, 26, 215 .

[28] Y. Shen, A. Bax, J. Biomol. NMR 2007, 38, 289.

[29] X. P. Xu, D. A. Case, J. Biomol. NMR 2001, 21, 321.

[30] N. A. Lakomek, K. F. Walter, C. Fares, O. F. Lange, B. L. de Groot, H. Grubmuller, R. Bruschweiler, A. Munk, S. Becker, J. Meiler, C. Griesinger, J. Biomol. NMR 2008, 41, 139.

[31] S. L. Chang, N. Tjandra, J. Magn. Reson. 2005, 174, 43.

[32] A. Knocks, H. Weingartner, J. Phys. Chem. B 2001, 105, 3635. 